\title{
The root transcriptome for North American ginseng assembled and profiled across seasonal development
}

\author{
Di Wu ${ }^{1,2+}$, Ryan S Austin ${ }^{2 \dagger}$, Sijun Zhou ${ }^{1,2}$ and Dan Brown ${ }^{1,2,3^{*}}$
}

\begin{abstract}
Background: Ginseng including North American ginseng (Panax quinquefolius L.) is one of the most widely used medicinal plants. Its success is thought to be due to a diverse collection of ginsenosides that serve as its major bioactive compounds. However, few genomic resources exist and the details concerning its various biosynthetic pathways remain poorly understood. As the root is the primary tissue harvested commercially for ginsenosides, next generation sequencing was applied to the characterization and assembly of the root transcriptome throughout seasonal development. Transcripts showing homology to ginsenoside biosynthesis enzymes were profiled in greater detail.

Results: RNA extracts from root samples from seven development stages of North American ginseng were subjected to 454 sequencing, filtered for quality and used in the de novo assembly of a collective root reference transcriptome consisting of 41,623 transcripts. Annotation efforts using a number of public databases resulted in detailed annotation information for 34,801 (84\%) transcripts. In addition, 3,955 genes were assigned to metabolic pathways using the Kyoto Encyclopedia of Genes and Genomes. Among our results, we found all of the known enzymes involved in the ginsenoside backbone biosynthesis and used co-expression analysis to identify a number of candidate sequences involved in the latter stages ginsenoside biosynthesis pathway. Transcript profiles suggest ginsenoside biosynthesis occurs at distinct stages of development.

Conclusions: The assembly generated provides a comprehensive annotated reference for future transcriptomic study of North American ginseng. A collection of putative ginsenoside biosynthesis genes were identified and candidate genes predicted from the lesser understood downstream stages of biosynthesis. Transcript expression profiles across seasonal development suggest a primary dammarane-type ginsenoside biosynthesis occurs just prior to plant senescence, with secondary ginsenoside production occurring throughout development. Data from the study provide a valuable resource for conducting future ginsenoside biosynthesis research in this important medicinal plant.
\end{abstract}

Keywords: North American ginseng, Transcriptome, Next generation sequencing, Ginsenoside

\section{Background}

Ginseng belongs to species within the genus Panax (family Araliaceae) that comprises approximately 14 species of slow-growing perennial plants with fleshy roots [1]. North American ginseng (Panax quinquefolius L.) is native to eastern North America [2] and Panax

\footnotetext{
* Correspondence: dbrown@sbrc.ca

${ }^{\dagger}$ Equal contributors

'Western University, 1151 Richmond St, London, ON, Canada

${ }^{2}$ Agriculture and Agri-Food Canada, 1391 Sandford Street, London, ON, Canada

Full list of author information is available at the end of the article
}

species have been used for thousands of years to improve the overall health of human beings, as a remedy to promote vitality, assist the body functions, improve the immune system and protect against stress. It also has been recommended for years as a traditional medicine for a multitude of diseases such as cancer, inflammation, diabetes, cardiovascular ills and obesity [3-7] as well as being a recent source of natural extracts mass marketed as a cold prevention treatment [8].

Ginsenosides are considered to be the major bioactive compounds behind many of the claims of ginseng's

\section{Biomed Central}


health benefits; they are triterpenoid saponins found nearly exclusively in ginseng and have been the target of considerable research effort [9-11]. To date, more than 150 naturally occurring ginsenosides have been isolated from Panax species and most of them can be classified into two groups based on the skeleton of their aglycones, namely dammarane-type and oleanane-type $[12,13]$. The dammarane-type consists mainly of three varieties, classified according to their genuine aglycone moieties: 20Sprotopanaxadiol (PPD), 20S-protopanaxatriol (PPT), and ocotillol. Rb1, Rb2, Rc, Rd (PPDs) and Re and Rg1 (PPTs) are the most abundant six ginsenosides found in North American ginseng. Over 90\% of total ginsenoside content from North American ginseng belongs to these two groups $[14,15]$.

North American ginseng contains high levels of Rb1, $\mathrm{Rd}$ and Re ginsenosides-higher than those of Panax ginseng [16]. Ro is the only saponin of the oleanane-type ginsenoside, found as a minor component in North American ginseng [14]. Ginsenosides are biosynthesized via the mevalonate pathway [17]. Using expressed sequence tag analysis, it has been possible to identify several candidate genes encoding for the enzymes farnesyl diphosphate synthase (FPS) and squalene synthase (SQS), involved in the various biosynthetic steps from isopentenyl pyrophosphate and dimethylallyl pyrophosphate to squalene [18-20]. The cyclization of oxidosqualene is the branch point for the biosynthesis of ginsenosides and plant sterols. The common steps from acetyle-CoA to 2, 3-oxidosqualene have been widely studied [21]. The 2, 3-oxidosqualene cyclases (OSCs) that synthesize $\beta$-amyrin and dammarenediol-II $[22,23]$, as well as the Cyt P450 enzyme CYP716A47 that catalyses the formation of protopanaxadiol from dammarenediol-II during ginsenoside biosynthesis have been found in Panax ginseng [24]. However, the rest of the downstream pathways of ginsenoside biosynthesis remain largely unexplored.

Over the past several years, next-generation sequencing technologies have revolutionized the analysis of genomic information [25]. As applied to the transcriptome with RNAseq, it has been successfully used for transcript profiling, as well as SNP discovery in a number of plant species and has dramatically improved the efficiency and speed of gene discovery [26-28]. The application of 454 next generation sequencing technology has seen a rapid improvement in throughput, read length and accuracy in the past few years, with the GS FLX Titanium server used in this study able to generate one million reads with an average length of 400 bases with $99.5 \%$ accuracy [29]. Meanwhile, the analysis of transcriptomic data often relies on aligning reads to a reference which is often not feasible for non-model plant species in which little genomic research has been performed. We applied the transcriptome assembly program Trinity to the assembly of a high quality reference transcriptome for North American ginseng. Trinity has been shown to recover most expressed transcripts as full-length sequences, and is also able to resolve alternative isoforms and duplicated genes, outperforming other de novo assembly tools [30,31]. Our application to ginseng resulted in 41,623 ginseng root transcripts. We fully annotated $84 \%$ of these transcripts using sequence similarity searches and protein domain scanning with publicly available databases. In our results, we were able to identify predicted representatives for all of the known enzymes involved in the ginsenoside backbone biosynthesis and also profile their expression levels across seasonal development.

\section{Results}

Commercial production of ginseng usually results in harvests after 3 to 5 years. In this study, three-year-old roots were collected, washed, sorted for uniformity and overwintered and grown under simulated growing season conditions in the Biotron facility to minimize variation in environmental factors and soil pathogen infection. Root samples were collected over the fourthyear full growing season at seven development stages [32]: 1) budding - leaf emergence; 2) leaves - distinctive separation of leaf and stem; 3) flowering - plant in full flower; 4) green fruit set; 5) ripe fruit - fruit coloration fully red; 6) fruit drop - including early signs of leaf senescence (e.g., leaf curl) and 7) senescence - complete senescence of leaf and stem (the normal commercial harvest stage) (Figure 1).

\section{Sequencing and de novo assembly}

Tissue from the seven developing stages was collected from 4-year-old North American ginseng grown under controlled conditions in a growth chamber programmed to simulate southern Ontario, Canada commercial growing conditions (Additional file 1). Ginsenoside content levels for each development stage in American ginseng are listed in Table 1. Total RNA was isolated from the roots of each stage, and messenger RNA purified by Ambion Poly (A) Purist ${ }^{\text {tw }}$ mRNA Purification Kits. A half plate of 454 sequencing on the GS FLX Titanium platform was applied to each sample (http://www.454.com). This generated from 327 to $391 \mathrm{Mbp}$ of sequence for each stage with an average sequence length of 553 base pairs. All sequencing reads from the seven development stages were deposited in the NCBI (National Centre for Biotechnology Information) and can be accessed at the Sequencing Read Archive (SRA) with the accession numbers SRX247045, SRX247043, 


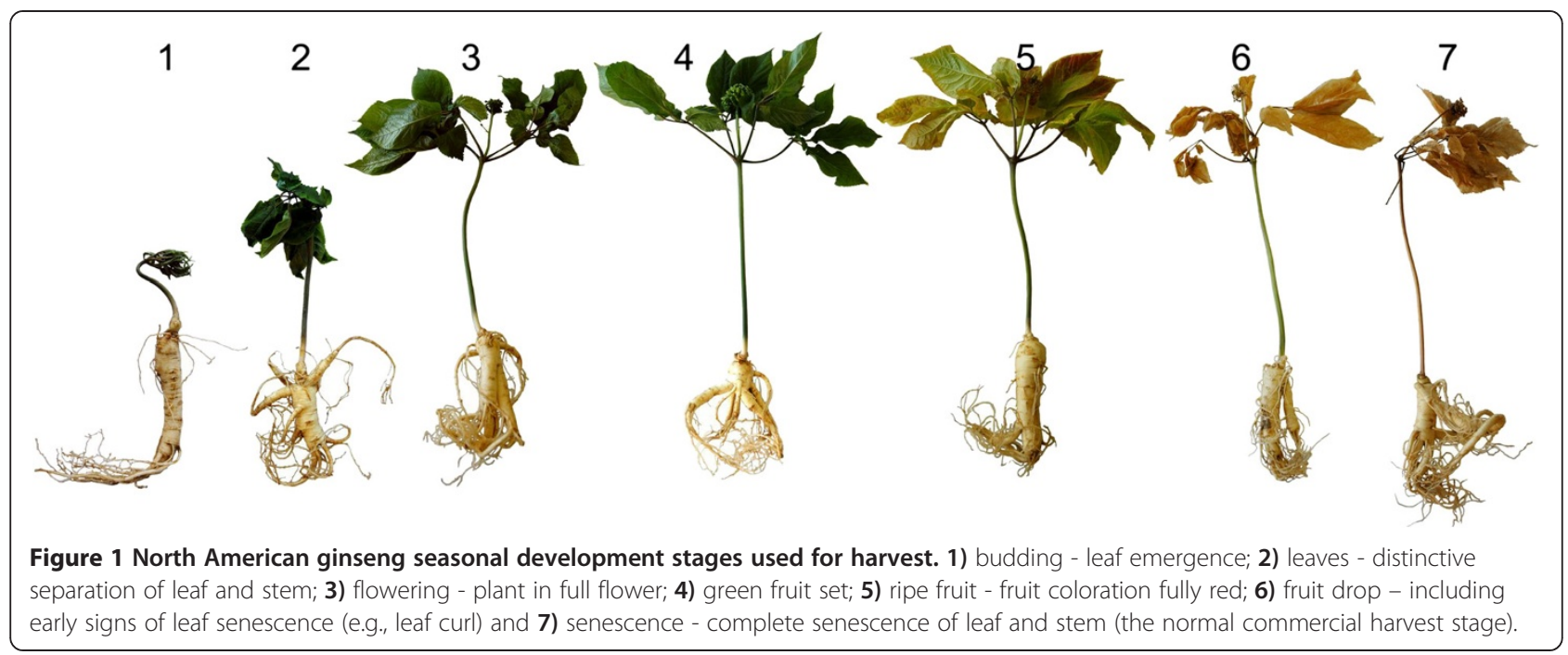

SRX247042, SRX247040, SRX247039, SRX247038 and SRX247037 for stages one through seven respectively.

Filtering of the sequence data before assembly included steps that removed plastid contaminants and adaptor sequences, as well as trimmed the base pair bias present in the first $15 \mathrm{bp}$ of the 5 ' end and low quality bases $(\mathrm{Q}<30)$ at the 3 ' end. Quality score distributions for reads in each stage before and after filtering are provided in Additional file 2. After filtering, 1,222,382 sequence reads remained for assembly with an average length of 348 base pairs (Table 2). Unfortunately, our poly-A purification step failed to effectively filter the abundant rRNA present and a large percentage of reads had to be filtered from each stage as contaminants arising from ribosomal or plastid RNA sequences. Interestingly, after quality trimming the reads, a disproportionate amount of sequences with lengths of approximately 340 and 410 base pairs were found (Figure 2a). This is presumably an artefact of trends in sequence quality drop off at specific points during sequencing.

High quality reads from all stages were combined and provided to the transcriptome assembly program Trinity. The resulting assembled $37.3 \mathrm{Mb}$ of transcriptome contained 21,340 genes or 41,623 transcripts when including the different gene isoforms Trinity is capable of returning. This number represents more than 8 times the number of North American ginseng sequences presently deposited in Genbank (as of March, 2013). Transcript lengths ranged from 300 to 7,719 base pairs with an average length of $896 \mathrm{bp}$ and the majority of transcripts ranging between $500 \mathrm{bp}$ and $2 \mathrm{~Kb}$ in size (Figure 2b). Almost half of all genes assembled (49\%) possessed at least one isoform, with a total of 20,283 splice variants identified by Trinity and $11 \%$ of genes possessing 6 or more splice variants (Figure 3). One gene (Pq315) possessed 96 different isoforms, although we felt this may have been an artefact of the assembly process.

In a similarity comparison to 5,018 Panax quinquefolius ESTs in Genbank, $87.82 \%$ were present in our assembly with strong significance $\left(E<\mathrm{e}^{-20}\right)$. When Genbank ESTs specifically derived from the Panax quinquefolius rhizome were considered, this number increased to $92.66 \%$, suggesting a high quality, comprehensive sampling of the root developmental transcriptome. To simplify identification and enable easy reference, all sequences in the assembly were assigned a unique identifier derived from the

Table 1 Ginsenoside contents of development stage among American ginseng ( $P$. quinquefolius) in Ontario

\begin{tabular}{|c|c|c|c|c|c|c|c|}
\hline \multirow[b]{2}{*}{ Stage } & \multicolumn{6}{|c|}{ Ginsenoside composition $^{\mathrm{a}}$ (ug/g, 75\% Ethanol extraction) } & \multirow[t]{2}{*}{ Total ginsenosides $(\mathrm{mg} / \mathrm{g}$} \\
\hline & Rg1 & $\operatorname{Re}$ & Rb1 & Rc & $\mathbf{R b}$ & Rd & \\
\hline 1 & 2251 & 30602 & 91611 & 7879 & 1072 & 15795 & 14.92 \\
\hline 2 & 1441 & 22318 & 116252 & 11024 & 2104 & 17338 & 17.05 \\
\hline 3 & 1273 & 19313 & 86478 & 7669 & 1185 & 15194 & 13.11 \\
\hline 4 & 2395 & 22863 & 82086 & 6042 & 965 & 11751 & 12.61 \\
\hline 5 & 1755 & 22408 & 73849 & 6452 & 994 & 7324 & 11.28 \\
\hline 6 & 2055 & 22268 & 85416 & 5970 & 964 & 6999 & 12.37 \\
\hline 7 & 1671 & 15019 & 94093 & 6083 & 1000 & 8523 & 9.11 \\
\hline
\end{tabular}

a: Samples were tested in duplicate and number of root samples tested in each stage. 
Table 2 Summary of cDNA sequencing results

\begin{tabular}{llllllll}
\hline & Stage 1 & Stage 2 & Stage 3 & Stage 4 & Stage 5 & Stage 6 & Stage 7 \\
\hline No. raw reads & 748,332 & 610,853 & 712,270 & 672,867 & 721,897 & 756,630 & 625,784 \\
Raw nt (Mb) & 390.6 & 327.8 & 372.2 & 361 & 368.4 & 388.2 & 327.2 \\
Raw avg. length (bp) & 522 & 537 & 728 & 537 & 510 & 513 & 523 \\
No. filtered reads & 159,812 & 173,693 & 132,038 & 149,483 & 191,274 & 173,273 & 242,359 \\
Filtered nt (Mb) & 52.6 & 63.9 & 44.1 & 52.7 & 65.3 & 59.0 & 90.5 \\
Filt. avg. length (bp) & 329 & 368 & 334 & 353 & 341 & 340 & 373 \\
\% in assembly & 58.5 & 62.6 & 62.3 & 64.2 & 60.1 & 62.0 & 61.2 \\
\hline
\end{tabular}
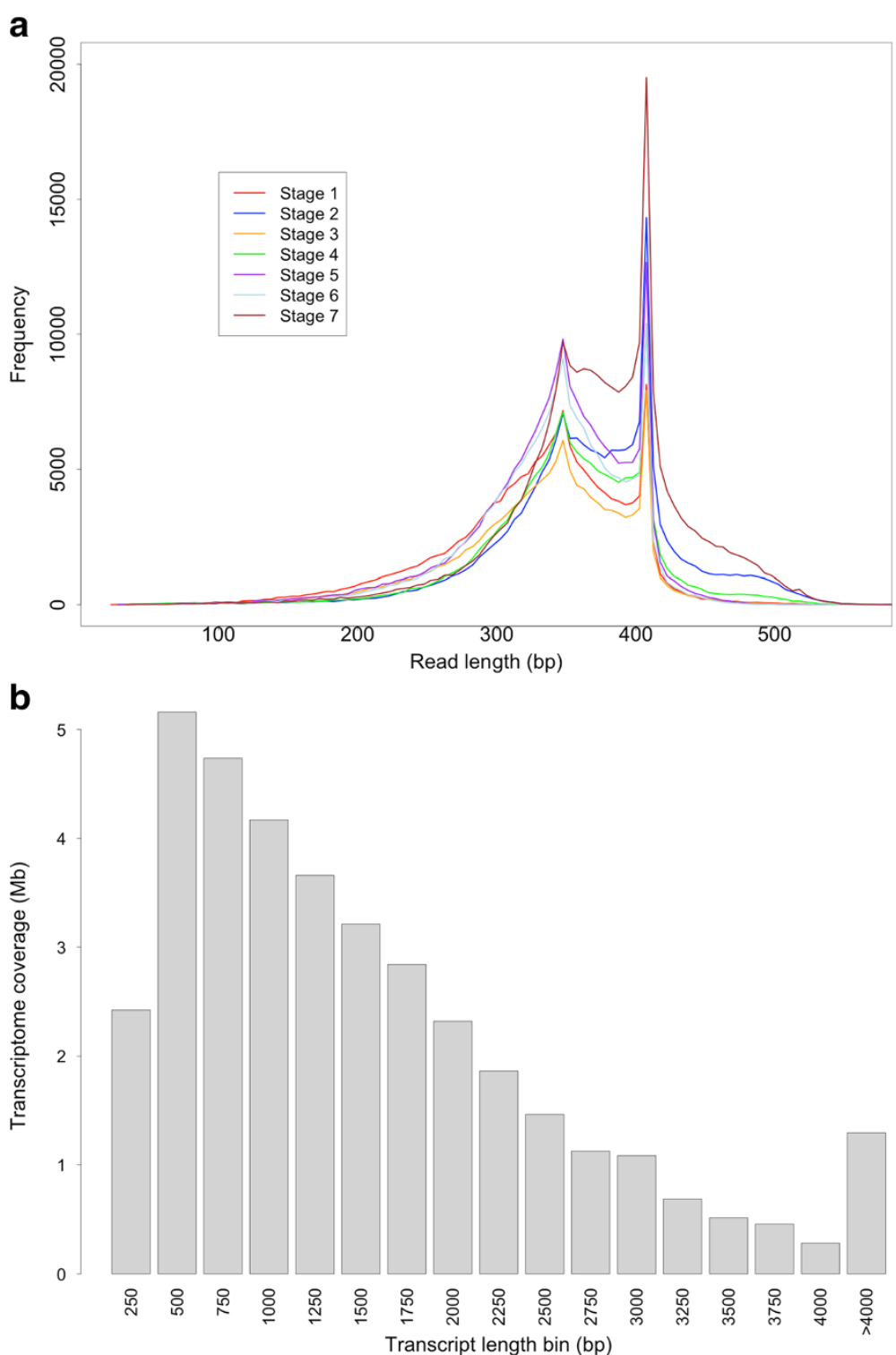

Figure 2 Filtered reads and assembled transcript length distributions. (a) Distribution of sequence read lengths used in transcriptome assembly from each of the seven developmental stages after quality filtering and trimming. (b) Histogram of assembled transcripts binned at $300 \mathrm{bp}$ intervals and the relative contribution of each bin to the overall transcriptome size. 


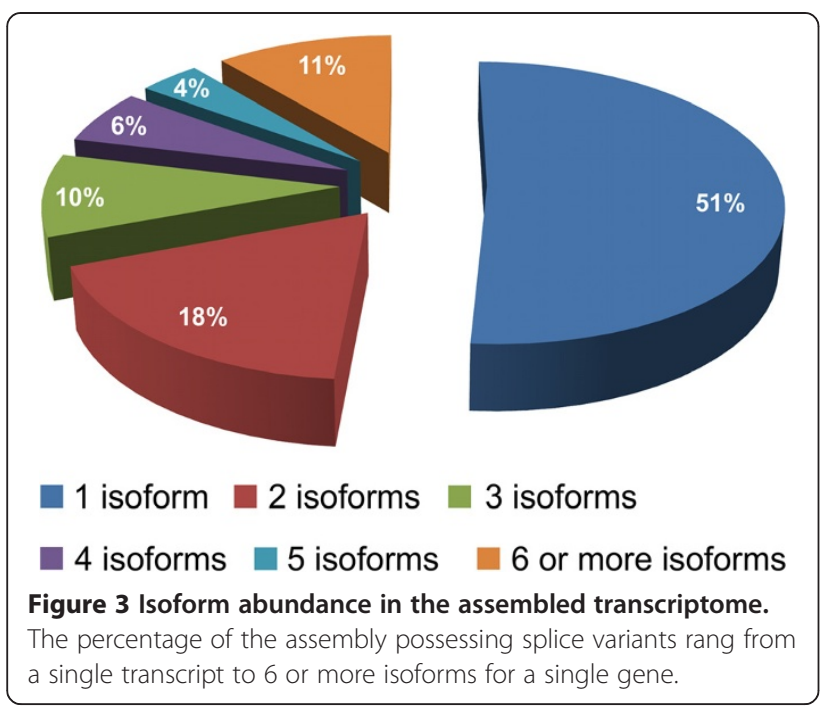

Trinity graph component and appended with a splice number that followed the form of "Pqx.y", where "Pq" stands for Panax quinquefolias, " $x$ " is the Trinity component number and " $y$ " is the splice variant number.

\section{Transcript annotation with public databases}

To facilitate as complete an annotation as possible for the assembly, sequence similarity searches [33] were performed against a collection 5,018 Ginseng ESTs from GenBank, the Arabidopsis genome (TAIR10), the uniProt Plant Protein Annotation Program (PPAP) database and GenBank's non-redundant (nr) protein database. In addition, protein domain scanning using hidden Markov models (HMMs) from Pfam were applied as well as the assignment of metabolic pathway information from the Kyoto Encyclopedia of Genes and Genomes (KEGG) database. Overall, these efforts annotated 83.6\% of transcripts in the assembly.

Comparison against the Genbank nr database yielded 33,366 hits $\left(\mathrm{E}<1 \mathrm{e}^{-10}\right)$, accounting for $80.16 \%$ of transcriptome sequences. A full $87.2 \%$ of these hits showed strong homology $\left(\mathrm{E}<\mathrm{e}^{-20}\right)$. As the $\mathrm{nr}$ database contains few ginseng proteins, we examined the different species with which homology was found. The majority of hits $(55.7 \%)$ were found to be against grape (Vitis vinifera), followed by castor oil plant (Ricinus communis) (14.5\%), black cottonwood poplar (Populus trichocarpa) (12.4\%), and soybean (Glycine max) (1.6\%). Similar results were found with searches against the Plant Protein Annotation Program (PPAP) database from Uniprot and the TAIR10 release of the Arabidopsis genome yielding $33,522(80.54 \%)$ and 30,990 (74.45\%) transcripts with significant hits $\left(\mathrm{E}<1 \mathrm{e}^{-10}\right)$ (Additional file 3).

As Arabidopsis is the most thoroughly annotated plant, sequence homology to Arabidopsis was also used to characterize the transcriptome based on Gene
Ontology (GO) information. GO annotation provides descriptions of gene products in terms of their associated molecular functions, cellular components, and biological processes. Using sequence homology to TAIR10, 14,537 GO terms were assigned to 24,110 sequences categorized into 80 functional groups. GO assignments were most frequently related to biological processes $(6,431)$, followed by cellular components $(12,489)$ and molecular function $(5,190)$ (Figure 4).

The assembly was scanned with protein domain HMM models from the Pfam database in order to catalogue any significant matches $\left(\mathrm{E}<\mathrm{e}^{-10}\right)$ to known protein domains. Overall, 32,277 HMMs were scanned against the assembly resulting in annotation for 21,263 transcripts possessing 17,266 different protein domains. This added annotation information to an additional 77 transcripts that had no hits in the prior homology searches. The most abundant domain found was the protein kinase domain, present in 1,310 transcripts. This is a similar number to the 1,719 kinases present in the Arabidopsis genome and not surprising, as protein kinases play a role in a multitude of cellular processes, including division, proliferation, apoptosis, and differentiation.

Finally, in order to assign metabolic information to our transcripts, the KAAS tool (http://www.genome.jp/ tools/kaas/) [34] was used to assign pathway information from the KEGG database. This resulted in a KEGG orthology (KO) number for 5,717 transcripts that possessed homology with metabolic enzymes in the KEGG database. The pathways most strongly represented in the results were protein processing in the endoplasmic reticulum, ubiquitin mediated proteolysis, nucleotide excision repair, arginine and proline metabolism, peroxisome, amino sugar and nucleotide sugar metabolism, proteasome, starch and sucrose metabolism and glycolysis/gluconeogenesis. All annotation information and significance scores were summarized and concatenated with transcript identifiers into a single line of annotation for each sequence in a Fasta formatted transcriptome file (Additional file 3).

\section{Expression profiles across development}

To evaluate the contribution of each stage of development to the reference transcriptome and profile the relative expression levels of transcripts throughout development, we mapped all high quality reads from each stage back to the assembled transcriptome using the BWA tool [35]. While this is useful in determining relative transcript abundance, it did have the unfortunate side effect of reporting unmapped reads for 6,113 transcripts. Investigating further, it was found that nearly all unmapped transcripts were involved in alternative splicing and of the shortest transcripts in the assembly (mean length: $414 \mathrm{bp}$ ). We presume that this is a 


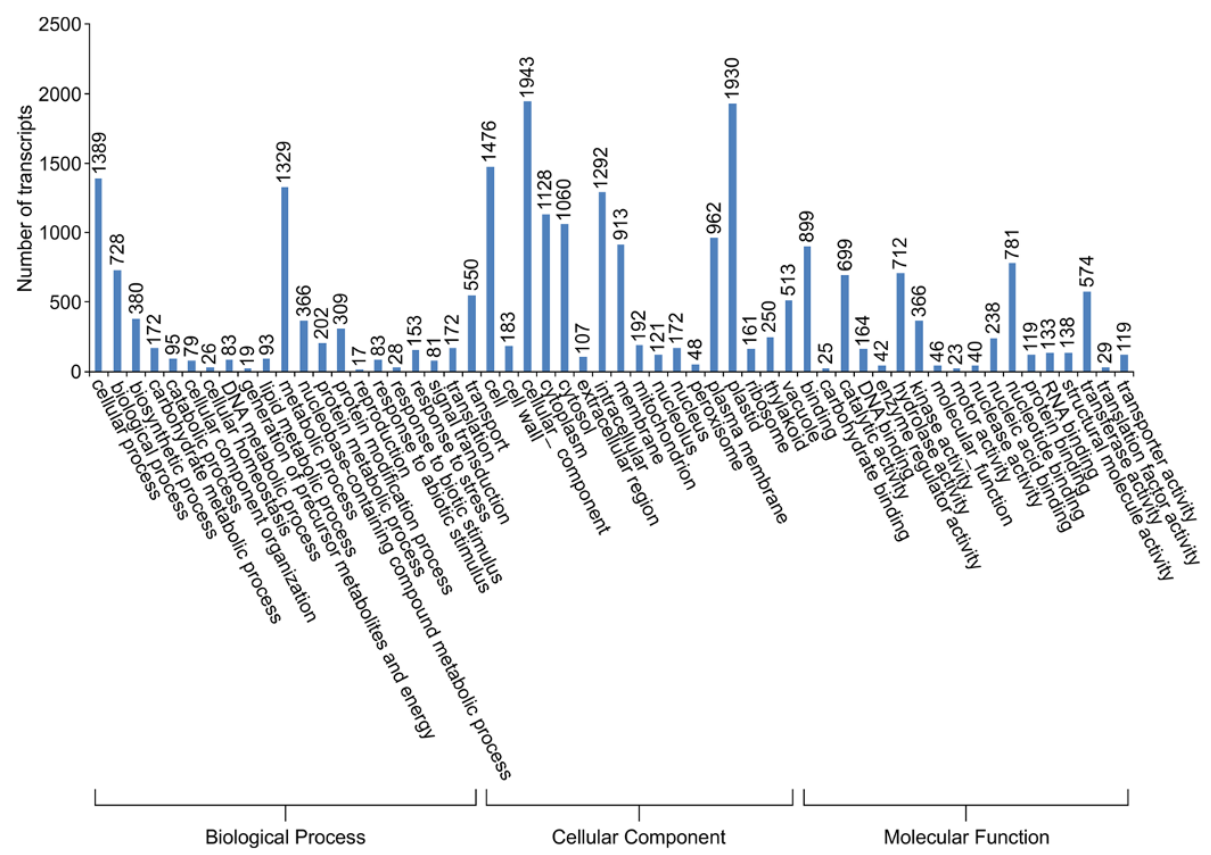

Figure 4 Functional classification of North American ginseng based on gene ontology categories. Gene ontology categories and classifications as identified by feeding the assembled transcriptome to the BLAST2GO web server.

Table 3 Top 20 most highly expressed transcripts in P. quinquefolius root transcriptome evaluated across all developmental stages

\begin{tabular}{|c|c|c|}
\hline Unique ID & RPKM & Function \\
\hline $\mathrm{Pq00.2}$ & 50250.3 & Ribonuclease-like storage protein [Panax ginseng] \\
\hline Pq00.1 & 34703.4 & Ribonuclease-like storage protein [Panax ginseng] \\
\hline Pq250.4 & 14090.4 & Specific abundant protein-like protein 1 [Panax quinquefolius] \\
\hline Pq230.7 & 9875.59 & S-adenosylmethionine synthetase 2 [Arabidopsis thaliana] \\
\hline Pq411.1 & 9742.28 & Tonoplast intrinsic protein [Panax ginseng] \\
\hline Pq291.2 & 8594.7 & Methionine synthase [Hemp broomrape] \\
\hline Pq420.1 & 8176.25 & Major latex-like protein [Panax ginseng] \\
\hline Pq480.1 & 7957.75 & Ribosomal protein L15 [Populus balsamifera subsp. trichocarpa] \\
\hline Pq380.1 & 7692.05 & tRNA/rRNA methyltransferase (SpoU) family protein [Arabidopsis thaliana] \\
\hline Pq540.1 & 7655.38 & Thioredoxin H-type 1 [Arabidopsis thaliana] \\
\hline Pq140.1 & 7191.77 & Plasma membrane intrinsic protein PIP1-1 [Fraxinus excelsior] \\
\hline Pq323.5 & 7188.64 & Ascorbate peroxidase [Nicotiana tabacum] \\
\hline Pq140.3 & 6558.44 & Aquaporin [lris hollandica] \\
\hline $\mathrm{Pq} 470.11$ & 6270.28 & Actin 7 [Arabidopsis thaliana] \\
\hline Pq430.1 & 6038.08 & Translation initiation factor SUI1 family protein [Arabidopsis thaliana] \\
\hline Pq660.2 & 6003.21 & Nucleic acid-binding, OB-fold-like protein [Arabidopsis thaliana] \\
\hline Pq93.7 & 5982.31 & Dehydrin 4 [Panax ginseng] \\
\hline Pq740.1 & 5947.93 & $\beta$-amylase 3 [Arabidopsis thaliana] \\
\hline Pq400.1 & 5940.15 & DCD domain protein [Arabidopsis thaliana] \\
\hline Pq571.2 & 5831.33 & Catalase 2 [Arabidopsis thaliana] \\
\hline
\end{tabular}


consequence of the $\mathrm{k}$-mer $(\mathrm{k}=25)$ assembly process used by Trinity, perhaps creating isoform transcripts that are shorter than the long 454 reads from which they originally derive or possibly from read trimming by the alignment program. Nevertheless, expression information was obtained for over $85 \%$ of the transcriptome which comprised the longer contigs.

Counts for mapped reads were then used to generate RPKM (reads per kilobase transcript per million reads mapped) values for each putative transcript in each developmental stage. Of the most abundantly expressed predicted proteins, storage proteins topped the list, with a number of Panax ginseng annotated proteins: RNaselike major storage protein, specific abundant protein, tonoplast intrinsic protein, major latex-like protein and dehydrin 4 (Table 3).

We also examined the level of overlap in transcript expression occurring between major developmental stages of the plant. To limit the complexity in the number of comparisons needed, the seven developmental stages sampled were grouped into the broader developmental categories of budding (stages 1/2), flowering (stage 3), fruiting (stages 4/5) and senescence (stages 6/7). Overall, $26,681,17,990,26,162$ and 26,772 predicted transcripts showed positive expression in each category respectively. Each category possessed around two thousand predicted transcripts uniquely expressed during that stage of root development, with the exception of flowering, which had 621 specific transcripts. However, this is likely influenced by the fact that flowering is the only single stage group and thus represents a snapshot from a shorter period of developmental time compared to the other stages. Interestingly, the senescence stage had the largest number of predicted transcripts overlapping with other developmental stages. Altogether, a total of 13,074 transcripts were found to be expressed throughout all stages of development (Figure 5).

As ginsenosides are the ultimate compound of commercial interest in ginseng harvest, we sought to inventory all putative transcripts in the assembly that could be implicated in the synthesis of ginsenosides and examine their expression profiles across seasonal development. We therefore took all KEGG orthology numbers associated with enzymes in the mevalonate pathway of terpenoid backbone biosynthesis along with those from the sesquiterpenoid and triterpenoid biosynthesis pathways leading to chair-chair-chair-boat conformation triterpenoids (of which ginsenosides belong) and pulled all transcripts annotated with corresponding $\mathrm{KO}$ numbers in the assembly. This returned 14 predicted genes (43 transcripts including isoforms) annotated and identified by KEGG orthology. In addition, as there was no

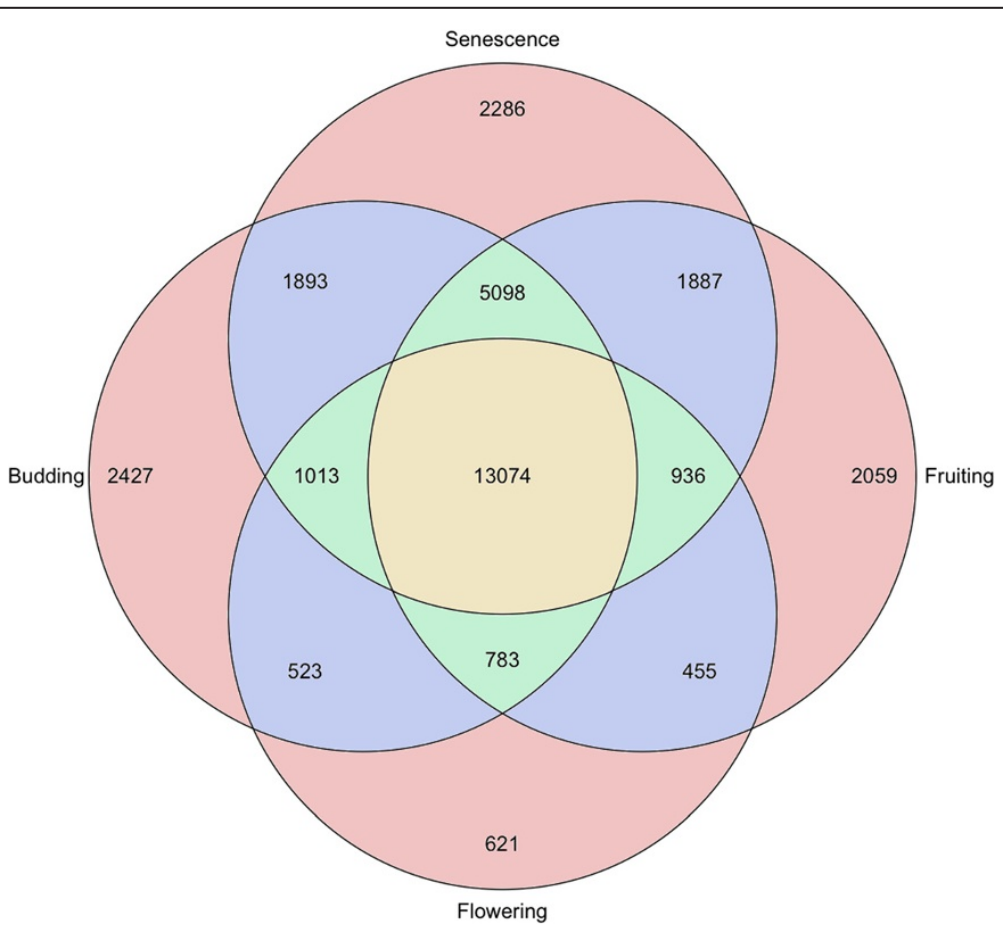

Figure 5 Venn diagram of transcript abundance across development. The 7 developmental stages sampled were grouped into four categories for a simplified comparison of transcript abundance: Budding - stages 1 \& 2; Flowering - stage 3; Fruiting - stages 4 \& 5; Senescence - stages 6 \& 7. RPKM values for transcript expression in each developmental category were used to establish transcript presence or absence and results compared. 
Table 4 Assembly genes implicated in ginsenosides biosynthesis

\begin{tabular}{|c|c|c|c|c|}
\hline Gene & Alias & $\mathrm{EC}$ & KO & Annotation \\
\hline Pq9090 & AACT & 2.3.1.9 & K00626 & Acetyl-CoA acetyltransferase \\
\hline $\mathrm{Pq} 24050$ & HMGS & 2.3.3.10 & K01641 & HMG-CoA synthase \\
\hline $\mathrm{Pq} 28460$ & HMGR & 1.1.1.34 & K00021 & HMG-CoA reductase (NADPH) \\
\hline $\mathrm{Pq} 48060$ & MK & 2.7.1.36 & K00869 & Mevalonate kinase \\
\hline Pq106070 & PMK & 2.7.4.2 & K00938 & Phosphomevalonate kinase, mvaK2 \\
\hline Pq40790 & MDD & 4.1.1.33 & K01597 & Mevalonate diphosphate decarboxylase \\
\hline Pq10790 & $|\mathrm{D}|$ & 5.3.3.2 & K01823 & |sopentenyl-diphosphate-isomerase \\
\hline Pq57960 & FPS/DMAPP & 2.5.1.1 & K14066 & Farnesyl diphosphate synthase/IPP-dimethylallyltransferase \\
\hline Pq25790 & FPS/DMAPP & 2.5.1.1.1/10 & K00787 & Farnesyl diphosphate synthase/IPP-dimethylallyltransferase \\
\hline Pq130580 & FPS/DMAPP & 2.5.1.1.1/10 & K13789 & Farnesyl diphosphate synthase/IPP-dimethylallyltransferase \\
\hline Pq44950 & FPS/DMAPP & 2.5.1.1.1/10 & K13789 & Farnesyl diphosphate synthase/IPP-dimethylallyltransferase \\
\hline $\mathrm{Pq} 28280$ & SQS & 2.5.1.21 & K00801 & Squalene synthase \\
\hline Pq129610 & SQE1 & 1.14.13.132 & K00511 & Squalene epoxidase 1 \\
\hline $\mathrm{Pq} 7190$ & SQE1 & 1.14.13.132 & K00511 & Squalene epoxidase 1 \\
\hline Pq41740 & DS & 4.2.1.125 & K15817 & Dammarenediol-II synthase \\
\hline Pq137240 & AS & & & $\beta$-amyrin synthase \\
\hline Pq133900 & AS & & & $\beta$-amyrin synthase \\
\hline Pq215750 & AS & & & $\beta$-amyrin synthase \\
\hline Pq104900 & AS & & & $\beta$-amyrin synthase \\
\hline Pq194830 & AS & & & $\beta$-amyrin synthase \\
\hline Pq75200 & P450 & & & Cytochrome P450, CYP73A5 \\
\hline Pq7360 & P450 & & & Cytochrome P450, CYP71B25 \\
\hline Pq9780 & P450 & & & Cytochrome P450, CYP75B1 \\
\hline Pq10460 & P450 & & & Cytochrome P450, CYP71A26 \\
\hline Pq142430 & P450 & & & Cytochrome P450 \\
\hline Pq108220 & P450 & & & Cytochrome P450, CYP710A1 \\
\hline Pq137680 & GT & & & UDP-Glycosyltransferase \\
\hline $\mathrm{Pq} 47050$ & GT & & & Glycosyltransferase, GAUT9 \\
\hline Pq177130 & GT & & & Glycosyltransferase, GAUT14 \\
\hline Pq117050 & GT & & & UDP-Glycosyltransferase \\
\hline Pq158770 & GT & & & a 1,4-Glycosyltransferase \\
\hline Pq10160 & GT & & & UDP-Glycosyltransferase, 71C4 \\
\hline Pq315* & GT & & & UDP-Glycosyltransferase \\
\hline
\end{tabular}

* This gene possessed 96 splice variants and may be an artefact of assembly.

appropriate assigned KEGG EC for $\beta$-amyrin synthase, five $\beta$-amyrin synthase annotated genes (6 transcripts including isoforms) were pulled for analysis using homology annotation in the assembly (Table 4).

Squalene synthase plays an important role as the precursor in backbone biosynthesis of the dammarenedioltype ginsenosides [36,37]. There are three previously reported squalene synthases in Panax ginseng [38] including squalene synthase (PgSS1), squalene synthase 1 (SQS1) and squalene synthase 2 (SQS2). SQS1 and SQS2 were also previously found in Panax quinquefolius
[39]. Although, we found 3 putative genes (i.e. Pq71210, Pq96200, Pq28280) with a significant squalene/phytoene synthase domain (Pfam: PF00494), only one (Pq28280) was annotated with KEGG orthology to SQS. This gene showed strong identity $(\mathrm{E}=0.0)$ with $A$. thaliana the SQS1 gene.

In North American ginseng, the majority of ginsenosides are known to be of the dammarene-type ginsenosides produced from protopanaxdiol and protopanaxtriol triterpenes. Dammarenediol-II produces protopanaxdiol and protopanaxtriol, and ginsenosides are thought to be 
synthesized from subsequent hydroxylation of these products by cytochrome P450 enzymes and glycosylation by glycosyltransferases (GTs) [22-24]. Our assembly contained 175 predicted transcripts annotated as Cytochrome P450s; with 63 of these possessing high similarity to P450 sequences from Panax ginseng and Panax notoginseng EST collections. Similarly, the assembly contained 164 predicted transcripts annotated as glycosyltransferases with 54 of these previously identified in Panax ginseng, Panax notoginseng and Panax quinquefolias. In order to identify potential candidates from these gene families that may be functioning in the latter stages of ginsenoside biosynthesis, we conducted a co-expression analysis with the transcript profiles for our putative dammarenediol synthase (DS) and squalene expoxidase (SQE) found immediately upstream in the ginsenoside biosynthesis pathway.

The expression profiles for the two strongest SQE (Pq129610.1) and DS (Pq41740.1) annotated transcripts in our assembly showed very high co-expression ( $\mathrm{r}>$ 0.89). This is not unexpected given that DS follows SQE in the biosynthesis pathway. We reasoned that candidate downstream P450 and glycosyltransferase genes may be similarly co-expressed. Co-expression analysis between all putative P450s and our predicted DS transcript identified 6 candidate P450s highly co-expressed with DS $(\mathrm{r}>0.85)$ across the 7 developmental stages sampled (Pq75200.2, Pq7360.1, Pq9780.1, Pq10460.1, Pq142430.1 and Pq108220.1). In the case of Pq75200.2, co-expression with DS was extremely high $(r>0.96)$. Similarly, 6 glycosyltransferases annotated transcripts were highly coexpressed with our predicted DS ( $\mathrm{r}>0.85$ ) (Pq137680.1, Pq47050.2, Pq177130.1, Pq117050.1, Pq158770.1, and Pq10160.3). As before, one transcript (Pq137680.1) showed extremely high co-expression $(r>0.94)$. Similar results were found in co-expression with the upstream SQE (data not shown). It should be noted that two splice variants from a single gene were also returned in the glycosyltransferase co-expression analysis. However, these transcripts derived from a gene (Pq315) for which 96 splice variants were identified by Trinity. As the annotation information for these 96 isoforms showed no strong consistency across isoforms, we concluded that this may be an artefact of the assembly, perhaps from the misinterpretation of a gene family, and were therefore dropped to simplify the analysis.

In order to provide biological validation to the 12 predicted transcripts showing coexpression with our putative DS gene, we conducted real-time PCR against the DS gene itself, the 6 putative glycosyltransferase genes and 6 putative cytochrome $\mathrm{P} 450$ genes across the final 3 stages of development. We were able to successfully amplify all 13 genes using RT-PCR analysis and confirmed strong coexpression between our predicted DS, 5 of the glycosyltransferase transcripts and 4 of the P450 transcripts (Figure 6). Additional file 4 lists the primers used in this analysis.

Before examining the relative expression profiles of our identified biosynthesis genes we sought to first look at the expression profiles for the entire transcriptome across seasonal development. RPKM values for all predicted transcripts were thus hierarchically clustered, and displayed in a heat map to create a transcriptome-wide display of developmental expression (Figure 7a). Many transcripts showed maximum expression within one or two specific stages of root development relative to other stages, with all stages of development possessing distinct clusters of genes showing dominant expression within that stage. Moreover, compared to other stages of development, senescence possessed a disproportionately large cluster of transcripts exhibiting a senescence-specific maximum in expression.

Expression profiles for all predicted genes and their isoforms implicated in ginsenoside biosynthesis (Figure 7b) as identified above were also hierarchically clustered and plotted in a heat map (Figure 7c). For representatives of downstream candidates, the most highly co-expressed P450 and glycosyltransferase transcripts identified in the co-expression analysis were also included in the collection. As seen with the entire transcriptome, many enzymes

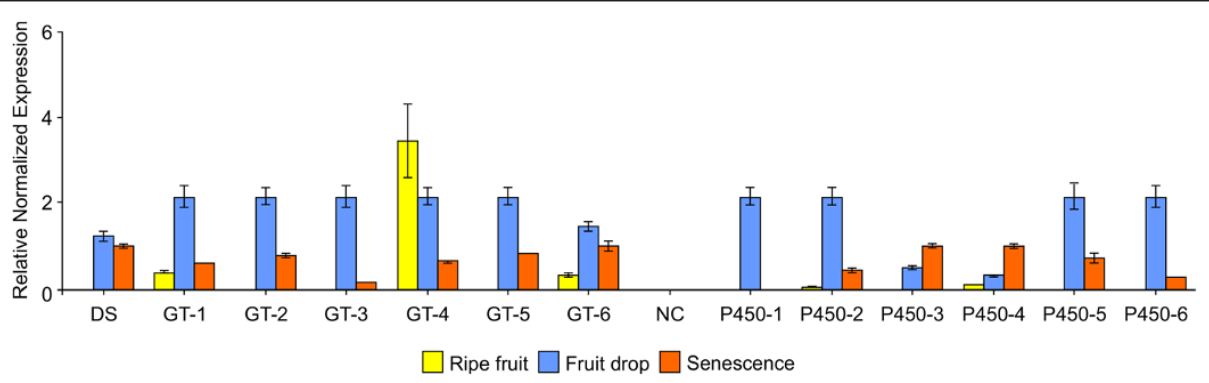

Figure 6 Real time PCR analysis. Real-time PCR results for 6 putative cytochrome P450 transcripts and 6 putative glycosyltransferase transcripts found to be highly co expressed $(r>0.9)$ with a predicted dammarenediol synthase (DS) evaluated across the last 3 development stages of ripe fruit, fruit drop and senescence. Corresponding unique transcript identifier numbers in the assembly are: DS: Pq41740.1; GT-1: Pq137680.1; GT-2: Pq177130.1; GT-3: Pq117050.1; GT-4: Pq158770.1; GT-5: Pq47050.2; GT-6: Pq10160.3; NC - negative control; P450-1: Pq75200.2; P450-2: Pq7360.1; P450-3: Pq9780.1; P450-4: Pq10460.1; P450-5: Pq142430.1; P450-6: Pq108220.1. 


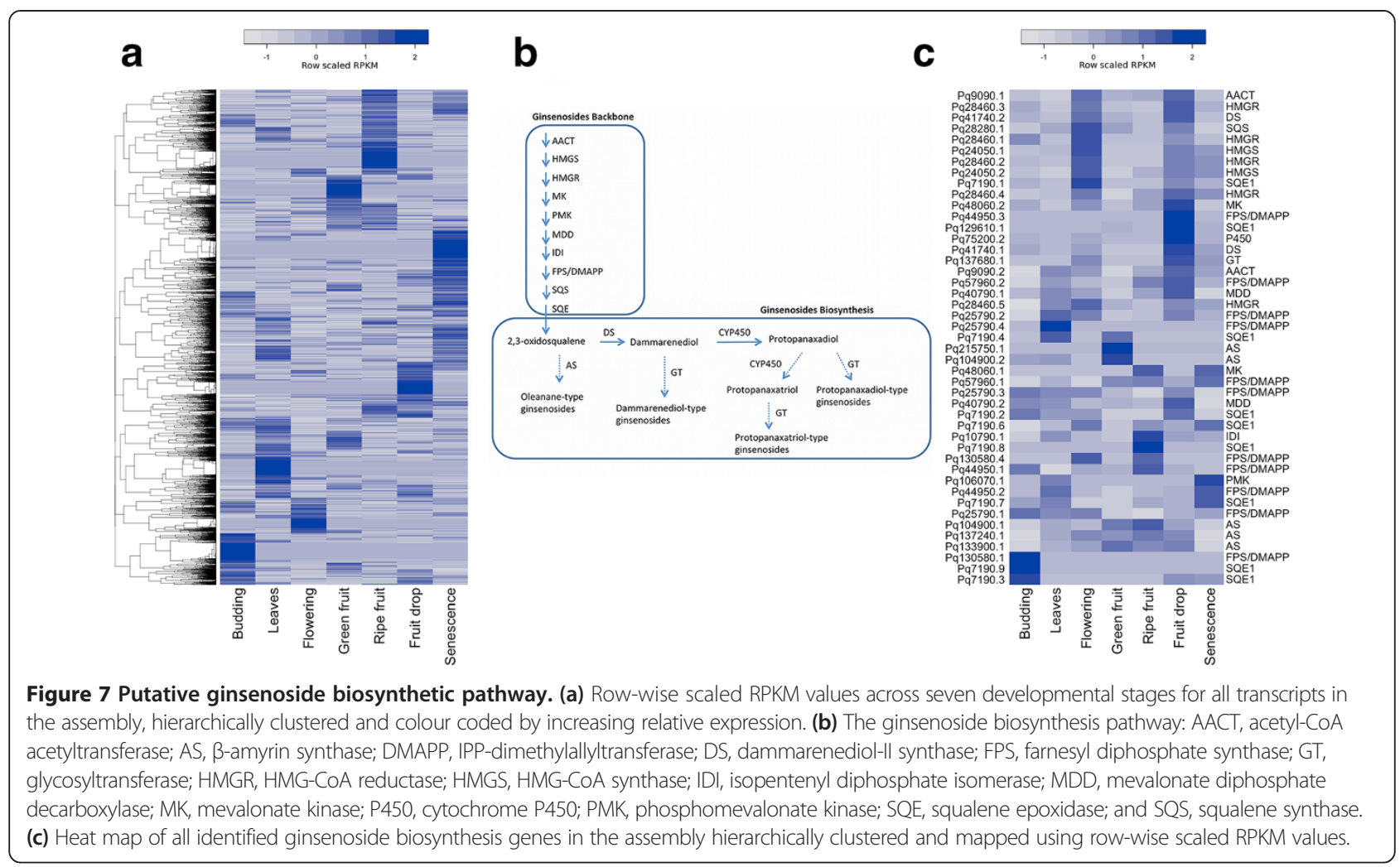

displayed abundant expression, specific to one or a few developmental stages relative to the other stages. To our surprise, stage 3 (Flowering) and stage 6 (Fruit drop) both possessed obvious clusters that encompassed putative representatives for almost all the enzymes in the biosynthesis pathway, suggesting that these two developmental stages are important points of ginsenoside biosynthesis in the developing plant. In the case of stage 7 , this makes intuitive sense considering the traditional time of ginseng harvest after the fruit drop stage and onset of leaf senescence. Interestingly, when different isoforms of genes were present, they tended to exhibit expression maxima in different stages of development. This was most apparent with the 9 different isoforms of SQE1 (Pq7190), for which at least one instance of an isoform with stage specific expression was found in all developmental stages except senescence stage 7 (Figure 7c). Of course, due to the computational limitations of assembly and mapping, both the isoforms identified and the expression profiles should be interpreted with caution without further in planta validation.

\section{Discussion}

Ginsenosides are found exclusively in the plant genus Panax and are viewed as the primary active compounds behind the claims of ginseng's health efficacy. The ginsenosides, also known as triterpenoids belong to the class of terpenoid saponines and are synthesized via the mevalonate pathway, which shares a common pathway with sterol. Oxidosqualene is a precursor common to the biosynthesis of both steroid and triterpenoids in higher plants [36]. The cyclization of 2, 3-oxidosqualene to oleanane and dammarane triterpenes is the first step in ginsenosides synthesis. The primary enzymes of triterpenoid saponions synthases are $\beta$-amyrin synthase (AS), and lupeol or dammarenediol-II synthase (DS). Putative orthologs for DS and AS were both identified in our assembly. $\beta$-amyrin synthase synthesizes oleanane-type triterpene and oleanane-type ginsenosides; however, they are found in only minor amounts in North American ginseng. On the contrary, over $90 \%$ of the total ginsenosides of North American ginseng belong to the dammarane-type. Interestingly, in our results, predicted AS transcripts appear most abundantly within developmental stages in which predicted DS enzymes are not abundant (i.e., flowering and fruiting). Moreover, they are not associated with the distinctive co-expression clusters of abundant putative ginsenoside biosynthesis genes as seen with DS in stages 3 and 6 . This potentially emphasizes the minor role of AS derived ginsenosides in North American ginseng. At the same time, the clusters of predicted biosynthesis enzymes during flowering and fruit drop highlight a potential significance of damamarane-type synthesis and suggest that flowering and fruit drop could be primary points of ginsenoside biosynthesis during seasonal development, with most emphasis on the latter. 
While we observed distinct stages of specific up-regulation for most predicted enzymes in the assembly, the presence of different isoforms with expression abundance specific to different stages of development is intriguing. In particular, our putative SQE1 possessed 9 different isoforms that showed stage specific expression abundance in all stages of development save stage 6 (fruit drop). Curiously, an alternate predicted SQE1 (Pq129610.1) with only a single isoform was strongly expressed in this stage. This seems to suggest that variation in alternative splicing may be a mechanism for producing varied forms of ginsenosides during seasonal development. Generally speaking, it also implies that alternative splicing may function as a means for directing variation in secondary metabolite production throughout the course of plant development. However, due to the inherent computational limitations involved in assembly and mapping, further analysis in the form of qPCR and associated metabolic assays is needed to prove or disprove any such hypotheses.

Of course, the assembly process will not be perfect with respect to isoform prediction and the transcripts themselves. There is a strong potential for misassembly in the form of merged gene families, close paralogs, or even alleles of the same gene being misreported as isoforms. While the comparison with Ginseng ESTs in Genbank is reassuring of the assembly quality, all predictions should be treated with caution in the absence of biological validation. Similarly, the mapping of reads to the assembly is limited by the presence of isoforms, as the actual point of origin for the read is confounded by the presence of potential multiple sources. This introduces a level of stochastic noise to the expression analysis that is mostly confined to genes with several isoforms.

That said, real-time PCR was able to validate the presence of a number of transcripts within expected developmental stages, as well as confirm their coexpression and upregulation within the fruit drop stage of development. Transcripts for a predicted DS gene, six putative P450s and six putative glycosyltransferases were all confirmed as present in planta and expression levels for four of the P450s and five of the glycosyltransferases confirm a tight coregulation with the predicted DS gene across the last stages of development as seen in our expression data. These predicted enzymes are thus strong candidates for controlling ginsenoside biosynthesis in the late stages of plant development.

While this study, as any next-generation sequencing study, would have benefited from an even larger amount of sequence data, additional sequencing over so many stages of development was unfortunately cost prohibitive. Nevertheless, the overall structure of the assembly with regard to number of genes, isoform frequency, length of transcripts and level of homology with existing EST libraries and annotation patterns identified among the transcripts is all very supportive and indicative of a strong representation of the biology. Overall, we believe the analysis benefited considerably from the use of the much longer reads that 454 sequencing is capable of generating. This added information translates into more reliable, longer, and complete transcripts, as well as more information for improved accuracy in the calling of alternative splicing among sequenced transcripts.

Recent work by Sun et al., found similar benefits to the use of 454 sequencing in the assembly of a transcriptome for North American ginseng [39]. Our work has expanded on these initial efforts through the generation of over six times the amount of high quality 454 reads sampled over seven stages of seasonal root development. This provides an unprecedented snapshot of North American ginseng root development and a means to comprehensively capture root expressed transcripts and their relative abundance through developmental time. In addition, we have also taken advantage of the recent Trinity assembly algorithm, which is designed specifically for assembling transcriptomes. Altogether, this provides the benefit of a high confidence, reliable assembly of putative transcripts that includes potentially valuable splice variant information. In the end, the combination of a fully annotated assembly along with corresponding expression data as sampled across developmental time has provided for an insightful look into the transcriptome of North American ginseng.

\section{Conclusions}

Ginseng is a valuable medicinal herb that is widely used in traditional medicine and has considerable commercial value. This study has focused on the ginseng root as it is the tissue almost exclusively used for medicinal applications and contains the majority of the commercially and medicinally valuable ginsenosides. Using extensive sampling of root tissue across seasonal development and nextgeneration sequencing we have generated a reliable North American ginseng transcriptome with extensive annotation and isoform information. Expression profiling of all ginsenoside backbone biosynthesis genes suggests that ginsenoside biosynthesis is tightly linked with the developmental stages of flowering and fruit drop. We anticipate the assembly and associated expression data should prove invaluable to those conducting transcriptomic and metabolomic research against Panax quinquefolius.

\section{Methods}

\section{Sample collection and preparation}

In the first week of October, three-year-old North American ginseng (Panax quinquefolius L.) was collected from the field of a cooperating commercial grower and member of the Ontario Ginseng Growers Association (http://ginsengontario.com/), near Delhi, Ontario, Canada. Plants were washed, sorted for size 
uniformity and placed in a peat-based growing medium (Promix BX, Priemer Tech Horticulture) filled containers in the Biotron facility at Western University (http://www.thebiotron.ca/). After a simulated over-wintering treatment at $4^{\circ} \mathrm{C}$ in the dark for 120 days, plants were transferred to a growth chamber in the first week of February under conditions which simulated the normal spring / summer field conditions for temperature, sunlight and humidity in the southern Ontario commercial growing region (Additional file 1). The four-year-old roots were collected at seven development stages (Figure 1), rinsed with cold water, chopped into small pieces, and immediately immersed in liquid nitrogen and stored at $-80^{\circ} \mathrm{C}$ until further processing.

The possibility of transcript "contamination" by bacterial and fungal pathogens was minimised by careful cleaning of the roots and growth in a peat-based commercial (Promix BX) growing medium. This medium contained a biostimulate (Bacillus pumilus) and mycorrhizae (Glomus intradices). Pathogens commonly found in Canada colonised on Panax roots [40] include: Phytophthora cactorum, Pythium ultimum, Rhizoctonia solani, Fusarium solani, F. oxysporum, F. aveaceum, F. equiseti and Cylindrocarpon destructons. A search of the final assembly annotation after blasting against the NR database showed no hits against these species.

\section{RNA extraction}

One gram of frozen root tissue was ground to a fine powder in liquid nitrogen and transferred into $10 \mathrm{ml}$ RNAzol $^{\oplus}$ RT reagent (Molecular Research Center, Inc). This was vortexed vigorously for $5 \mathrm{~min}$ to make a complete suspension before $4 \mathrm{ml}$ RNase free water was added, incubated for $15 \mathrm{~min}$ (r.t.), $2 \mathrm{ml}$ bromo-chloroform (Sigma) added and centrifuged at $4^{\circ} \mathrm{C} 12,000 \mathrm{rpm}$ $(14,900 \mathrm{~g})$ for $15 \mathrm{~min}$. The supernatant was transferred into a new tube, $10 \mathrm{ml}$ phenol chloroform (25:24:1, pH6.8, Ambion) added, mixed, and centrifuged at $4^{\circ} \mathrm{C} 12,000 \mathrm{rpm}$ $(14900 \mathrm{~g})$ for $15 \mathrm{~min}$. The supernatant was transferred to a new tube and $3 \mathrm{ml}$ isopropanol plus $3 \mathrm{ml} 1.2 \mathrm{M} \mathrm{NaCl}$ added to precipitate total RNA. The mixture was incubated $15 \mathrm{~min}$ (r.t.), spun at $12000 \mathrm{rpm}(14,900 \mathrm{~g})$ for $15 \mathrm{~min}$ at $4^{\circ} \mathrm{C}$, and the supernatant discarded. $10 \mathrm{ml}$ of $75 \%$ ethanol was added to the pellet, vortexed to mix and then centrifuged for $10 \mathrm{~min}$ at 8,000 rpm (6630 g). The supernatant was discarded and the pellet resuspended with $3 \mathrm{ml}$ nuclease free water $\left(65^{\circ} \mathrm{C}\right)$. An equal volume of phenol: chloroform (25:24:1, pH 6.8) was added, the mixture vortexed and then centrifuged for $15 \mathrm{~min}$ at $12,000 \mathrm{rpm}$ $(14,900 \mathrm{~g})$. The supernatant was mixed with 0.1 volume $5 \mathrm{M}$ ammonium acetate and 2.5 volumes $100 \%$ ethanol. This was placed at $-20 \mathrm{C}$ overnight, or quickly frozen in either ethanol or dry ice, or in a $-80^{\circ} \mathrm{C}$ freezer for $30 \mathrm{~min}$. RNA was recovered by centrifugation at $\geq 12,000 \times \mathrm{g}$ for
$30 \mathrm{~min}$ at $4^{\circ} \mathrm{C} .1 \mathrm{~mL}$ of $70 \%$ ethanol was added to the pellet and the tube vortexed. The RNA was then pelleted by microcentrifugation at $12,000 \mathrm{rpm}(14,900 \mathrm{~g})$ for $10 \mathrm{~min}$ at $4^{\circ} \mathrm{C}$ and the pellet dissolved in $50 \mathrm{ul}$ nuclease free water. Extracted total RNA was cleaned using an RNeasy Mini Kit (QIAGEN).

\section{Sample preparation and sequencing}

Total RNA preparations were poly-A enriched prior to sequencing using a Poly (A) purist ${ }^{\mathrm{TM}}$ magnetic mRNA purification kit (Ambion). Isolated mRNA was qualified and quantified using an Agilent RNA 6000 pico kit on an Agilent 2000 Bioanalyser. Approximately 600-800 ng of isolated mRNA of each sample was sent to the DNA Technologies Laboratory at the National Research Council Canada for analysis. Samples were converted into cDNA using a cDNA Rapid Library Preparation Method (Roche) and sequenced on a GS FLX sequencer.

\section{Assembly and annotation}

Sequencing reads were filtered for contaminating plastid and ribosomal RNA sequences by comparison of all reads with a file of potential contaminants using BLAST $\left(\mathrm{E}<1 \mathrm{e}^{-10}\right)$. Custom Perl scripts were then used to remove any adaptor sequences, a base pair bias artefact from sequencing present in the first $15 \mathrm{bp}$ of the $5^{\prime}$ end and low quality bases $(\mathrm{Q}<30)$ at the 3 ' end. Filtered reads from all stages were concatenated together and fed to the Trinity assembler with a k-mer length of 25 and minimum transcript length of $300 \mathrm{bp}$. Similarity searches for annotating transcripts were performed using the BLAST blastn algorithm against Ginseng ESTs from Genbank, UniProt PPAP and TAIR10_pep_20101214_updated databases, and the blastx algorithm against Genbank nr. The Plant Protein Annotation Program (PPAP) database was built from the concatenation of the 'sprot' and 'trembl' files for plants downloaded from Uniprot (ftp.uniprot.org). KEGG pathway information was assigned to all transcripts using the KAAS KEGG Automatic Annotation Server (www.genome.jp/ tools/kaas/) [41]. Gene ontology information was assigned based on sequence similarity with Arabidopsis using the Blast2Go server (www.blast2go.org). Protein domain scanning was performed using the 32,273 HMM models contained in the PFAM A/B databases [42] and the hmmer tools [43]. Annotation data was processed and integrated into the final transcriptome reference using custom Perl scripts and UNIX tools. Transcript identifiers were generated from a concatenation of the species initials (Pq), the Trinity component and subcomponent identifier numbers, followed by a period and splice variant number.

\section{Expression profiling and visualization}

PCR duplicates were removed from filtered reads for each stage using Samtools before mapping reads against the 
assembled reference transcriptome using BWA (Q $>30)$. Reads were permitted to map to multiple places but only a single mapping using in downstream analysis. Investigation revealed that, presumably due to the very long read lengths, the vast majority of multiply mapped reads mapped to isoforms of the same gene. Reads with a map quality $>20$ were pulled and counted for each transcript using Samtools [44]. The reads per kilobase of transcript per million reads mapped (RPKM) value was then calculated for each transcript in each developmental stage using $\mathrm{R}$ (Additional file 5). Relative distance between RPKM values was assessed using Pearson correlation coefficients (PCC) and the transcript distance matrix clustered using divisive hierarchical clustering before visualization in a heat map that scaled RPKM expression values row-wise to a mean of zero and standard deviation of one using a Z-score. Co-expression between individual transcripts was assessed using PCC between RPKM values across all seven stages of development sampled.

\section{Real time PCR analysis}

After digestion with DNase I, approximately $1 \mu \mathrm{g}$ of total RNA from stage 5 ripe fruit, stage 6 fruit drop and stage 7 senescence were converted into first-strand cDNA via the reverse-transcription reaction with random hexamer primers (New England Biolabs) and SuperScript III Reverse Transcriptase Kit (Invitrogen). The cDNA products were then diluted 10 -fold with nuclease free deionized water before use as a template in real-time PCR. Specific cDNAs were amplified by SsoFast EvaGreen Supermix (Bio-Rad) in a volume of 10 ul. The reaction mixture contained $5 \mathrm{ul}$ SsoFast EvaGreen Supermix, $5 \mathrm{uM}$ each of the forward and reverse primers, and $4 \mu \mathrm{l}$ of template cDNA. PCR amplification was performed at the annealing temperature of $60^{\circ} \mathrm{C}$ with the CFX96 Real-Time PCR Detection System (Bio-Rad) according to the manufacturer's instructions. Relative transcript abundances were calculated by the comparative cycle threshold method with GAPDH as an internal standard, using the Bio-Rad CFX Manager software (Version 3). Primer pairs for RT-PCR were designed based on online software (http://www.ncbi.nlm.nih.gov/tools/primer-blast/) and are listed in Additional file 4.

\section{Additional files}

Additional file 1: Growth chamber condition settings for plant material.

Additional file 2: Quality score distribution of reads from all stages. Additional file 3: Panax quinquefolius annotated transcriptome in Fasta format.

Additional file 4: Primer pairs for RT-PCR analysis.

Additional file 5: RPKM values for all transcripts in transcriptome across seven stages.

\section{Competing interests}

The authors declare that they have no competing interests.

\section{Authors' contributions}

DW performed sample collection, RNA extraction, RT PCR, data analysis and drafted the manuscript. RA supervised DW in bioinformatic work, performed bioinformatic analysis of the data and helped write and edit the manuscript SZ helped with plant preparation and sample collection; DB conceived the study, supervised DW and SZ, coordinated the project design, and edited the manuscript. All authors read and approved the final manuscript.

\section{Acknowledgements}

This work was funded by the Ontario Research Foundation Research Excellence Program through the Ontario Ginseng Innovation and Research Consortium and supported by Agriculture and Agri-Food Canada. The authors thank Dr. Andrew Sharp for the service of cDNA library construction and 454 sequencing, and Dr. Chao Sun for ginseng genomic research consultation. The authors are also grateful to laboratory assistant Marysia Latoszek-Green and Jo Ann Stebbing for the help with the experiments and to the advice of several anonymous reviewers for their helpful suggestions on improving the manuscript.

\section{Author details}

${ }^{1}$ Western University, 1151 Richmond St, London, ON, Canada. ${ }^{2}$ Agriculture and Agri-Food Canada, 1391 Sandford Street, London, ON, Canada.

${ }^{3}$ Canadian Centre for Agri-food Research in Health and Medicine (CCARM), St. Boniface Hospital, 351 Taché Avenue, Winnipeg, MN, Canada.

Received: 17 April 2013 Accepted: 6 August 2013

Published: 19 August 2013

\section{References}

1. Case MA, Flinn KM, Jancaitis J, Alley A, Paxton A: Declining abundance of American ginseng (Panax quinquefolius L.) documented by herbarium specimens. Biol Conserv 2007, 134:22-30.

2. Nadeau I, Olivier A: The biology and forest cultivation of American ginseng (Panax quinquefolius L.) in Canada: a review. Can J Plant Sci 2003, 83:877-891.

3. Izzo AA: Phase II clinical trial reveals the excellent safety profile of Panax quinquefolius (American ginseng). Focus Alternat Complement Ther 2009, 14:24-25.

4. Tsao R, Liu Z: Exploration and characterization of bioactive phytochemicals in native Canadian plants for human health. Can J Plant Sci 2007, 87:1045-1053.

5. Wang CZ, Mehendale SR, Yuan CS: Commonly used antioxidant botanicals: active constituents and their potential role in cardiovascular illness. Am J Chinese Med 2007, 35:543-558.

6. Li B, Wang CZ, He TC, Yuan CS, Du W: Antioxidants potentiate American ginseng-induced killing of colorectal cancer cells. Cancer Lett 2010, 289:62-70.

7. Poddar K, Kolge S, Bezman L, Mullin GE, Cheskin L: Nutraceutical supplements for weight loss: a systematic review. Nutr Clin Pract 2011 26:539-552.

8. Predy GN, Goel V, Lovlin R, Donner A, Stitt L, Basu TK: Efficacy of an extract of North American ginseng containing poly-furanosyl-pyranosyl-saccharides for preventing upper respiratory tract infections: a randomized controlled trial. CMAJ 2005, 173:1043-1048.

9. Schlag EM, Mclntosh MS: Ginsenoside content and variation among and within American ginseng (Panax quinquefolius L.) populations. Phytochemistry 2006, 67:1510-1519.

10. Ko SK, Cho OS, Bae HM, Sohn UD, Im BO, Cho SH, Chung SH, Lee BY: Change of ginsenoside composition of various American ginseng roots. J Appl Biol Chem 2009, 52:198-201.

11. Kim SK, Park JH: Trends in ginseng research in 2010. J Ginseng Res 2011, 35:389-398.

12. Smith RG, Caswell D, Carriere A, Zielke B: Variation in the ginsenoside content of American ginseng, Panax quinquefolius L., roots. Can J Bot 1996, 74:1616-1620.

13. Chen CF, Chiou WF, Zhang JT: Comparison of the pharmacological effects of Panax ginseng and Panax quinquefolium. Acta Pharmacol Sin 2008, 29:1103-1108. 
14. Christensen LP: Ginsenosides. Chemistry, Biosynthesis, Analysis, and Potential Health Effects. Adv Food Nutr Res 2008, 55:1-99.

15. Qi LW, Wang CZ, Yuan CS: Ginsenosides from American ginseng: chemical and pharmacological diversity. Phytochemistry 2011, 72:689-699.

16. Asafu-Adjaye EB, Wong SK: Determination of ginsenosides (Ginseng Saponins) in dry root powder from panax ginseng, panax quinquefolius, and selected commercial products by liquid chromatography: Interlaboratory study. J AOAC Int 2003, 86:1112-1123.

17. Liang $Y$, Zhao S: Progress in understanding of ginsenoside biosynthesis. Plant Biology 2008, 10:415-421.

18. Choi DW, Jung J, Ha YI, Park HW, In DS, Chung HJ, Liu JR: Analysis of transcripts in methyl jasmonate-treated ginseng hairy roots to identify genes involved in the biosynthesis of ginsenosides and other secondary metabolites. Plant Cell Rep 2005, 23:557-566.

19. Luo ZY, Liu SP, Chen XH, Ruan Y, Luo JQ, Wen B, Liu CL, Hu WX: Spatio-temporal expression pattern of six novel candidate genes in ginsenoside biosynthesis from Panax ginseng C. A. Meyer. J Integr Plant Biol 2005, 47:622-631.

20. Kim YS, Han JY, Lim S, Choi YE: Ginseng metabolic engineering: regulation of genes related to ginsenoside biosynthesis. J Med Plant Res 2009, 3:1270-1276.

21. Wang J, Gao WY, Zhang J, Zuo BM, Zhang LM, Huang LQ: Advances in study of ginsenoside biosynthesis pathway in Panax ginseng C. A. Meyer. Acta Physiol Plant 2012, 34:397-403.

22. Kushiro T, Shibuya M, Ebizuka Y: $\beta$-Amyrin synthase. Cloning of oxidosqualene cyclase that catalyzes the formation of the most popular triterpene among higher plants. Eur J Biochem 1998, 256:238-244.

23. Tansakul P, Shibuya M, Kushiro T, Ebizuka Y: Dammarenediol-II synthase, the first dedicated enzyme for ginsenoside biosynthesis, in Panax ginseng. FEBS Lett 2006, 580:5143-5149.

24. Han JY, Kim HJ, Kwon YS, Choi YE: The Cyt P450 enzyme CYP716A47 catalyzes the formation of protopanaxadiol from dammarenediol-II during ginsenoside biosynthesis in Panax ginseng. Plant Cell Physiol 2011, 52:2062-2073

25. Nagalakshmi U, Waern K, Snyder M: RNA-seq: a method for comprehensive transcriptome analysis. Curr Protoc Mol Biol 2010, 89:1-4.

26. Marguerat S, Bähler J: RNA-seq: from technology to biology. Cell Mol Life Sci 2010, 67:569-579.

27. Asmann $\mathrm{YW}$, Wallace MB, Thompson EA: Transcriptome profiling using next-generation sequencing. Gastroenterology 2008, 135:1466-1468.

28. Marguerat S, Wilhelm BT, Bähler J: Next-generation sequencing: applications beyond genomes. Biochem Soc Trans 2008, 36:1091-1096.

29. Soares AR, Pereira PM, Santos MAS: Next-generation sequencing of miRNAs with Roche 454 GS-FLX technology: steps for a successful application. Methods Mol Biol 2012, 822:189-204.

30. Martin JA, Wang Z: Next-generation transcriptome assembly. Nat Rev Genet 2011, 12:671-682.

31. Grabherr MG, Haas BJ, Yassour M, Levin JZ, Thompson DA, Amit I, Adiconis $X$, Fan L, Raychowdhury R, Zeng $Q$, et al: Full-length transcriptome assembly from RNA-Seq data without a reference genome. Nat Biotechnol 2011, 29:644-652.

32. Wills RBH, Du XW, Stuart DL: Changes in ginsenosides in Australian-grown American ginseng plants (Panax quinquefolium L.). Aust J Exp Agr 2002, 42:1119-1123.

33. Altschul SF, Gish W, Miller W, Myers EW, Lipman DJ: Basic local alignment search tool. J Mol Biol 1990, 215:403-410

34. Ogata H, Goto S, Sato K, Fujibuchi W, Bono H, Kanehisa M: KEGG: Kyoto encyclopedia of genes and genomes. Nucleic Acids Res 1999, 27:29-34.

35. Li H, Durbin R: Fast and accurate short read alignment with BurrowsWheeler transform. Bioinformatics 2009, 25:1754-1760

36. Haralampidis K, Trojanowska M, Osbourn AE: Biosynthesis of triterpenoid saponins in plants. Adv Biochem Eng/Biotechnol 2002, 75:31-49.

37. Han JY, In JG, Kwon YS, Choi YE: Regulation of ginsenoside and phytosterol biosynthesis by RNA interferences of squalene epoxidase gene in Panax ginseng. Phytochemistry 2010, 71:36-46.

38. Kim TD, Han JY, Huh GH, Choi YE: Expression and functional characterization of three squalene synthase genes associated with saponin biosynthesis in panax ginseng. Plant Cell Physiol 2011, 52:125-137.

39. Sun C, Li Y, Wu Q, Luo H, Sun Y, Song J, Lui EMK, Chen S: De novo sequencing and analysis of the American ginseng root transcriptome using a GS FLX Titanium platform to discover putative genes involved in ginsenoside biosynthesis. BMC Genomics 2010, 11:262.
40. Punja ZK: Fungal pathogens of American ginseng (Panax quinquefolius) in British Columbia. Can J Plant Pathol 1997, 19:301-306.

41. Moriya Y, Itoh M, Okuda S, Yoshizawa AC, Kanehisa M: KAAS: an automatic genome annotation and pathway reconstruction server. Nucleic Acids Res 2007, 35:182-185. Web Server issue

42. Finn RD, Mistry J, Tate J, Coggill P, Heger A, Pollington JE, Gavin OL, Gunasekaran P, Ceric G, Forslund K, et al: The Pfam protein families database. Nucleic Acids Res 2009, 38:211-222. Database.

43. Johnson LS, Eddy SR, Portugaly E: Hidden Markov model speed heuristic and iterative HMM search procedure. BMC Bioinformatics 2010, 11:431.

44. Li H, Handsaker B, Wysoker A, Fennell T, Ruan J, Homer N, Marth G, Abecasis $G$, Durbin R: The Sequence Alignment/Map format and SAMtools. Bioinformatics 2009, 25:2078-2079.

doi:10.1186/1471-2164-14-564

Cite this article as: Wu et al:: The root transcriptome for North American ginseng assembled and profiled across seasonal development. BMC Genomics 2013 14:564.

\section{Submit your next manuscript to BioMed Central and take full advantage of:}

- Convenient online submission

- Thorough peer review

- No space constraints or color figure charges

- Immediate publication on acceptance

- Inclusion in PubMed, CAS, Scopus and Google Scholar

- Research which is freely available for redistribution

Submit your manuscript at www.biomedcentral.com/submit

C BioMed Central 